\title{
Managerial Decision scoring with Stochastic Programming on Selected Scaling Measures of Likert's Format
}

\author{
P. Tirupathi Rao ${ }^{1}$ and E. S. V. Narayana Rao ${ }^{2}$ \\ ${ }^{I}$ Dept. of Statistics, Pondicherry University, Puducherry - 605014, India. \\ ${ }^{2}$ Dept. of Statistics \& Computer Applications, Agricultural College, A.N.G.R. Agricultural University, Mahanandi- \\ 518502, A.P., India.
}

\begin{abstract}
Measuring the quality on quantitative formats is the most essential task where the attention of researchers is strongly desired. Modelling the scaling measures based on various formats is highly essential to explore several statistical devices. In this paper we have developed some stochastic models for the scaling measures similar to Likert's format. Bivariate stochastic processes in the context of spreadsheet experimentation were considered to develop these models. Mathematical formulae for several statistical measures of decision scores are derived for multiple choice decision items. The modelling is extended to formulate optimization programming problems with the objectives of maximising the expected decision score and also to minimise the volatility in decision score. The decision parameters namely, number of optimal decision assignments can be derived through the developed Non-Linear Programming Problem (NLPP). Sensitivity analysis is carried out with reference to optimal managerial decision scores.
\end{abstract}

Key Words: Stochastic modelling, optimisation programming, decision scores, scaling measures, bivariate stochastic process.

\section{Introduction}

Quantification of qualitative traits is the most essential task to get the parameters of the problem under study. Statistical approach of hypothesis verification is a valid scientific approach as it converts non-parametric inferences to a parametric inference. Adopting psychometric tools for measuring the managerial issues is need of the hour. Measuring the numerical value for a characteristic phenomenon has many limitations because of the complexity due to volatility of research tools of quantitative research. It is customary to use the ordinal scaling formats in measuring the performance of manager's program as they are mostly on categorical.

Invention of random variable and its relevance of measuring uncertainty is a break through to study the experimental process. Development of suitable stochastic models for the categorical and ordinal data will help in identification of data patterns in any experimental process. This study has developed some Bivariate stochastic models and also to derive the related probability distribution for measuring the performance of a manager. Quantifying the performance based on the descriptive/characteristic information is more complicated when compared to categorical/ordinal information. However, if the study orientation is on the objective of statistical investigation, then the methodology should be in quantitative formats.

Likert scale or semantic scale will measure the respondent's information on categorical scale as it converts the attributes into variables. Most of the research problems have been dealt with qualitative approach and in case study orientation. In those cases, the researchers are simply confined to make a simple analysis because of the nonavailability of proper mathematical models. Conversion of a general problem into a mathematical problem will always helpful in exploring the estimators of the population parameters by providing suitable formulae.

Mathematical modelling on scaling measures particularly like Likert's scale has opened a new area of research and has many interesting challenges. There is a lot of scope on modelling the scaling measures. Tirupathi Rao (2013) has initiated the modelling of the Likert's scaling measures using stochastic processes. An application of bivariate Bernoulli stochastic process has been considered for modelling the said scales. He has derived four stochastic models and the formulae for measuring four central moments. The study has also focused on developing simple and weighted probability models for bivariate spreadsheet experimentation.

Tirupathi Rao et.al. (2013) have also developed a stochastic programming for optimal decision making through scaling measuring while considering his previous works. They considered an environment of simple bivariate Bernoulli probability distribution, for designing managerial decision making and performance appraisals. The programming problem of maximising the expected decision score of a manager at different feasible constraints is developed and analysed it through simulated numerical data. 
Very little research work has been reported in this area. Hence, there is a good scope of pursuing research in this area for getting many managerial decision supporting systems. It has motivated the researchers to design some stochastic programming problems on the mentioned objectives.

In this study, the researchers have developed two stochastic optimisation programming problems with the objectives of maximising the average decision making score and minimising the volatility score. Constraints are formulated by incorporating several feasible issues.

\section{Stochastic Model And Optimisation Programming Problem}

For developing the model, the researchers have considered the context of managers' performance study basing on their decision making abilities. The decision scores have been measured with the bivariate stochastic process. Let the decision maker has ' $\mathrm{k}$ ' types of assignments where he want to make the decision basing on maximum expected decision score with minimum volatility.

Let there be ' $\mathrm{m}$ ' number of decision items in the process, each measured on ' $\mathrm{n}$ ' points of ascending ordered categorical scale. While making a decision he may or may not consider all the decision items. Further, different decision items have either with equal weight or with unequal weight. Therefore an uncertain situation prevails in selecting the decision item. While opting the scoring point for a specific decision item, he has to discriminate the scoring point based on the available information to him. Hence there is again uncertainty on opting the scaling point. Both the above phenomena leads to do the experimentation of "selecting proper decision item on suitable scale" is stochastic in nature.

Let $\mathrm{p}_{\mathrm{ij}}$ be the probability of choosing $\mathrm{i}^{\text {th }}$ decision item and awarding $\mathrm{j}^{\text {th }}$ score point to the selected item, where $i=1,2, \ldots \ldots \ldots ., m ; j=1,2, \ldots \ldots \ldots ., n$.

$\mathrm{p}_{\mathrm{ij}}=1$; when $\mathrm{j}^{\text {th }}$ score point is opted for $\mathrm{i}^{\text {th }}$ decision item ;

$\mathrm{p}_{\mathrm{ij}}=0$; when $\mathrm{j}^{\text {th }}$ score point is not opted for $\mathrm{i}^{\text {th }}$ decision item.

If the decision items are listed in rows and score points are listed in columns then row sums are equal to one. i.e. $\sum_{j=1}^{n} p_{i j}=1, \forall \mathrm{i}=1,2, \ldots \ldots . ., \mathrm{m}$; as $\mathrm{p}_{\mathrm{ij}}=1$ for $\mathrm{i}$ th row.

In more detail, there is no possibility of getting $\mathrm{p}_{\mathrm{ij}}=1$ at more than one cell in a row.

\section{Stochastic Model For Decision Score With Equal Weights Of Listed Items}

A decision making process usually considered with multiple decision items and the decision score is calculated by considering either all or partial decision items. However, in practice the decision maker may confine to a finite number of decision items among the proposed list. Once the number of decision items in the list was finalised, he may give equal weightage to the selected decision items. Let there be ' $\mathrm{m}$ ' decision items in the final list and they are all equally weighted. In this model, a bivariate discrete probability distribution is proposed with the following assumptions.

Let $\mathrm{q}_{\mathrm{ij}(1)}$ be the probability of opting $\mathrm{j}^{\text {th }}$ scale point for the $\mathrm{i}^{\text {th }}$ decision item when the decision items have the equal weightage. It is defined as

$$
\mathrm{q}_{\mathrm{ij}(1)}=\frac{p_{i j} w_{1}}{\sum_{i=1}^{m} w_{i}} \text {; where } \mathrm{w}_{\mathrm{i}}=\text { weight of } \mathrm{i}^{\text {th }} \text { decision item to be considered in the decision making process. As all wi's }
$$

are equally weighted, let $\mathrm{w}_{\mathrm{i}}=\mathrm{c}$ (constant), which implies $\mathrm{q}_{\mathrm{ij}(1)}=\mathrm{p}_{\mathrm{ij}} / \mathrm{m}$. $\mathrm{q}_{\mathrm{ij}(1)}$ will be acted as joint probability function such that

$$
\mathrm{q}_{\mathrm{ij}(1)} \geq 0 \forall \mathrm{i}=1,2, \ldots \ldots . . \mathrm{m} ; \quad \mathrm{j}=1,2, \ldots \ldots \ldots ., \mathrm{n} .
$$

$$
\sum_{i=1}^{m} \sum_{j=1}^{n} q_{i j(1)}=1
$$

Let $\mathrm{q}_{\mathrm{i} .(1)}$ be the marginal probability of $\mathrm{i}^{\text {th }}$ study item such that

$$
\mathrm{q}_{\mathrm{i} .(1)}=\sum_{j=1}^{n} q_{i j(1)} \geq 0 ; \text { for } \mathrm{i}=1,2, \ldots . ., \mathrm{m}
$$


(ii) $\quad \sum_{i=1}^{m} q_{i .(1)}=1$

Let $\mathrm{q}_{\mathrm{j}(1)}$ be the marginal probability of $\mathrm{j}^{\text {th }}$ scale point such that

$$
\begin{aligned}
& \text { (i) } \mathrm{q}_{\mathrm{j}(\mathrm{j}(1)}=\sum_{i=1}^{m} q_{i j(1)} \geq 0 ; \text { for } \mathrm{j}=1,2, \ldots ., \mathrm{n} \\
& \text { (ii) } \sum_{j=1}^{n} q_{. \mathrm{j}(1)}=1
\end{aligned}
$$

Let $\mathrm{k}_{\mathrm{i}}$ be the score component of $\mathrm{i}^{\text {th }}$ study item such that $\mathrm{k}_{\mathrm{i}}=\sum_{j=1}^{n} j p_{\mathrm{ij}}$ where ' $\mathrm{j}$ ' is the scale/score point. Let $\mathrm{S}_{\mathrm{w} 1}$ be the decision making score with equal weightage of decision items. As per Tirupathi Rao (2013), the over all decision score is,

$$
E\left(S_{w_{1}}\right)=\sum_{i=1}^{m}\left(\sum_{j=1}^{n} j P_{i j}\right)\left(\sum_{j=1}^{n} q_{i j(1)}\right)
$$

and the overall volatility score is,

$$
V\left(S_{w_{1}}\right)=\sum_{i=1}^{m}\left(\sum_{j=1}^{n} y_{j} P_{i j}\right)^{2}\left(\sum_{j=1}^{n} q_{i j(1)}\right)-\left(\sum_{i=1}^{m}\left(\sum_{j=1}^{n} y_{j} P_{i j}\right)\left(\sum_{j=1}^{n} q_{i j(1)}\right)\right)^{2}
$$

\section{Programming Problem Of Model 2.1}

The objective of the programming problem is to explore the decision variables such as the number of decision assignments belong to various categories. This sort of operational management is required to the agencies where they develop the decision support systems for deciding the optimal performing number of assignments.

Let the organization have ' $\mathrm{k}$ ' types of assignments based on which the decision making exercise such as finding the decision variables like optimal number of assignments is to be done, where $\mathrm{k}=1,2,3, \mathrm{r}, \mathrm{r} ; \mathrm{r}$ is the maximum number of different assignments. Let $X_{k}$ be the number of decision assignments in the $\mathrm{k}^{\text {th }}$ category of an organization. Let $\mathrm{C}_{\mathrm{k}}$ be the expected decision score on $\mathrm{k}^{\text {th }}$ category per assignment.

Let $Z=\sum_{k=1}^{r} C_{k} X_{k}$ which implies $Z=\sum_{k=1}^{r}\left(\sum_{i=1}^{m}\left(\sum_{j=1}^{n} j \cdot p_{i j}\right)\left(\sum_{j=1}^{n} q_{i j(1)}\right)\right) * X_{k}$ be the total expected decision score on all ' $r$ ' types of decision assignments. Since $\mathrm{Z}$ is the expected decision score, the objective is to maximize Z.

Let $\mathrm{T}_{\mathrm{k}}$ be the expected decision score for one assignment in the $\mathrm{k}^{\text {th }}$ category. The expected decision score for $\mathrm{X}_{\mathrm{k}}$ assignments in the $\mathrm{k}^{\text {th }}$ category is $\mathrm{T}_{\mathrm{k}} * \mathrm{X}_{\mathrm{k}}$. Therefore the overall expected score for all types of ' $\mathrm{r}$ ' assignments is $\sum_{k=1}^{r} T_{k} * X_{k}$. Usually the organization shall handle the decision making activities based on the expected score of each category of assignments and also on overall expected score of ' $r$ ' assignments. Let $\mathrm{S}_{\mathrm{k}}$ be the minimum targeted decision score based on which the $\mathrm{k}^{\text {th }}$ category of assignment is to be assessed, then the expected score should not be less than the specified score. Therefore, the constraints on the targeted score may be $\mathrm{E}\left(\mathrm{T}_{\mathrm{k}^{*}} \mathrm{X}_{\mathrm{k}}\right) \geq \mathrm{S}_{\mathrm{k}}$; which implies that $\sum_{i=1}^{m}\left(\sum_{j=1}^{n} j \cdot p_{i j}\right)\left(\sum_{j=1}^{n} q_{i j(1)}\right) * X_{k} \geq S_{k}$; for k=1,2,3, r; Further, we consider a constraint that 
the overall decision score based on all ' $\mathrm{r}$ ' categories. Let $\sum_{k=1}^{r} S_{k}=S$, then the constraint is $\sum_{k=1}^{r}\left(\sum_{i=1}^{m}\left(\sum_{j=1}^{n} j \cdot p_{i j}\right)\left(\sum_{j=1}^{n} q_{i j(1)}\right) * X_{k}\right) \geq S$

While achieving the objective, the decision maker has to observe the volatility score on decision making processes. Let $\mathrm{V}\left(\mathrm{T}_{\mathrm{k}}\right)$ be the variability of ' $\mathrm{k}$ 't ' type of one assignment. Then the variability for $\mathrm{X}_{\mathrm{k}}$ number of assignments is $\mathrm{V}\left(\mathrm{T}_{\mathrm{k}^{*}} \mathrm{X}_{\mathrm{k}}\right)$. Further, a healthy decision making procedure of $\mathrm{k}^{\text {th }}$ type requires the maximum restriction on the variability. Let $\mathrm{V}_{\mathrm{k}}$ be the maximum desired variability for $\mathrm{X}_{\mathrm{k}}$ number of assignments in the $\mathrm{k}^{\text {th }}$ category.

Therefore the overall variability for the $\mathrm{k}^{\text {th }}$ category should not exceed the specified level, i.e. $\mathrm{V}_{\mathrm{k}}$. Hence $\mathrm{V}\left(\mathrm{T}_{\mathrm{k}} * \mathrm{X}_{\mathrm{k}}\right) \leq \mathrm{V}_{\mathrm{k}}$, which implies that

$$
\left(\sum_{i=1}^{m}\left(\sum_{j=1}^{n} j \cdot p_{i j}\right)^{2}\left(\sum_{j=1}^{n} q_{i j(1)}\right)-\sum_{i=1}^{m}\left(\sum_{j=1}^{n} j \cdot p_{i j}\right)\left(\sum_{j=1}^{n} q_{i j(1)}\right)^{2}\right) * X_{k} \leq V_{k} ; \text { for } \mathrm{k}=1,2, \ldots . \mathrm{r} . \quad \text { Further, we consider }
$$

a constraint that the overall variability on decision score based on all ' $r$ ' categories. Let $\sum_{k=1}^{r} V_{k}=V$, then the constraint is

$\sum_{k=1}^{r}\left(\sum_{i=1}^{m}\left(\sum_{j=1}^{n} j \cdot p_{i j}\right)^{2}\left(\sum_{j=1}^{n} q_{i j(1)}\right)-\sum_{i=1}^{m}\left(\sum_{j=1}^{n} j \cdot p_{i j}\right)\left(\sum_{j=1}^{n} q_{i j(1)}\right)^{2}\right) * X_{k} \leq V$.

The decision variables under study are non negative. Hence $X_{k} \geq 0$. Therefore the overall optimization programming problem is

Maximize $Z=\sum_{k=1}^{r}\left(\sum_{i=1}^{m}\left(\sum_{j=1}^{n} j \cdot p_{i j}\right)\left(\sum_{j=1}^{n} q_{i j(1)}\right)\right) * X_{k}$

subject to the constraints

$$
\begin{aligned}
& \sum_{i=1}^{m}\left(\sum_{j=1}^{n} j \cdot p_{i j}\right)\left(\sum_{j=1}^{n} q_{i j(1)}\right) * X_{k} \geq S_{k} ; \text { for } \mathrm{k}=1,2, \ldots \mathrm{r} ; \\
& \left(\sum_{i=1}^{m}\left(\sum_{j=1}^{n} j \cdot p_{i j}\right)^{2}\left(\sum_{j=1}^{n} q_{i j(1)}\right)-\sum_{i=1}^{m}\left(\sum_{j=1}^{n} j \cdot p_{i j}\right)\left(\sum_{j=1}^{n} q_{i j(1)}\right)^{2}\right) * X_{k} \leq V_{k} \quad \text { for } \mathrm{k}=1,2, \ldots . \mathrm{r} ; \\
& \sum_{k=1}^{r}\left(\sum_{i=1}^{m}\left(\sum_{j=1}^{n} j \cdot p_{i j}\right)\left(\sum_{j=1}^{n} q_{i j(1)}\right) * X_{k}\right) \geq S \\
& \sum_{k=1}^{r}\left(\sum_{i=1}^{m}\left(\sum_{j=1}^{n} j \cdot p_{i j}\right)^{2}\left(\sum_{j=1}^{n} q_{i j(1)}\right)-\sum_{i=1}^{m}\left(\sum_{j=1}^{n} j \cdot p_{i j}\right)\left(\sum_{j=1}^{n} q_{i j(1)}\right)^{2}\right) * X_{k} \leq V ;
\end{aligned}
$$

and $\mathrm{X}_{\mathrm{k}} \geq 0$; for $\mathrm{k}=1,2, \ldots \mathrm{r}$

\section{Stochastic Model For Decision Score With Unequal Weights of Listed Items}


Let $\mathrm{q}_{\mathrm{ij}}$ be the probability of opting $\mathrm{j}^{\text {th }}$ score point for $\mathrm{i}^{\text {th }}$ study item, defined as $q_{i j(2)}=\frac{P_{i j} w_{i}}{\sum_{i=1}^{m} w_{i}}$ where $\mathrm{w}_{\mathrm{i}}$ is a variable i.e. $\mathrm{w}_{\mathrm{i}} \neq \mathrm{c} ; \mathrm{P}_{\mathrm{ij}}=1$; when $\mathrm{j}^{\text {th }}$ score point is being opted for the $\mathrm{i}^{\text {th }}$ study item; and $P_{i j}=0$; when $j^{\text {th }}$ score point is not being opted for the $\mathrm{i}^{\text {th }}$ study item; for $\mathrm{j}=1,2, \ldots ., \mathrm{n} ; \mathrm{i}=1,2, \ldots \ldots \mathrm{m}$ $q_{i .(2)}=\sum_{j=1}^{n} q_{i j(2)}$ is the marginal probability of $\mathrm{i}^{\text {th }}$ study item and $q_{. j(2)}=\sum_{i=1}^{m} q_{i j(2)}$ is the marginal probability of $\mathrm{j}^{\text {th }}$ score point. For the given joint distribution, $\sum_{j=1}^{n} q_{. j(2)}=\sum_{i=1}^{m} q_{i .(2)}=1$. Let $\quad k_{i}=\sum_{j=1}^{n} j P_{i j}$ be the score component of $\mathrm{i}^{\text {th }}$ study item. $\mathrm{y}_{\mathrm{j}}$ is the score point at $\mathrm{j}^{\text {th }}$ ordinate.

Let $\mathrm{S}_{\mathrm{w} 2}$ be the decision making score with unequal weightage of decision items. As per Tirupathi Rao (2013), the overall decision score is,

$$
E\left(S_{w_{2}}\right)=\sum_{i=1}^{m}\left(\sum_{j=1}^{n} j P_{i j}\right)\left(\sum_{j=1}^{n} q_{i j(2)}\right)
$$

and the overall volatility score is,

$$
V\left(S_{w_{2}}\right)=\sum_{i=1}^{m}\left(\sum_{j=1}^{n} j P_{i j}\right)^{2}\left(\sum_{j=1}^{n} q_{i j(2)}\right)-\left(\sum_{i=1}^{m}\left(\sum_{j=1}^{n} j P_{i j}\right)\left(\sum_{j=1}^{n} q_{i j(2)}\right)\right)^{2}
$$

\section{Programming Problem Of Model 2.2}

The objective of this programming problem is to explore the decision variables such as the number of different decision assignments belong to various categories. This sort of operational management is required to the agencies where they develop the decision support systems for deciding the optimal performing number of assignments.

Let the organization have ' $\mathrm{k}$ ' types of assignments based on which the decision making exercise such as finding the decision variables like optimal number of assignments is to be obtained, where $\mathrm{k}=1,2,3,, \mathrm{r} ; \mathrm{r}$ is the maximum number of different assignments. Let $\mathrm{Y}_{\mathrm{k}}$ be the number of decision assignments in the $\mathrm{k}^{\text {th }}$ category of an organization. Let $\mathrm{D}_{\mathrm{k}}$ be the expected decision score on $\mathrm{k}^{\text {th }}$ category per assignment.

Let $Z=\sum_{k=1}^{r} D_{k} Y_{k}$ which implies $Z=\sum_{k=1}^{r}\left(\sum_{i=1}^{m}\left(\sum_{j=1}^{n} j \cdot p_{i j}\right)\left(\sum_{j=1}^{n} q_{i j(2)}\right)\right) * Y_{k}$ be the total expected decision score on all ' $r$ ' types of decision assignments. Since $Z$ is the expected decision score, the objective is to maximize Z.

Let $\mathrm{T}_{\mathrm{k}}$ be the expected decision score for one assignment in the $\mathrm{k}^{\text {th }}$ category. The total expected decision score for $Y_{k}$ assignments in the $k^{\text {th }}$ category is $T_{k} * Y_{k}$. Therefore the overall expected score for all types of ' $r$ ' assignments is $\sum_{k=1}^{r} T_{k} * \mathrm{Y}_{k}$. Usually the organization shall handle the decision making activities based on the expected score of each category of assignments and also on overall expected score of ' $r$ ' assignments. Let $\mathrm{S}_{\mathrm{k}}$ be the minimum targeted decision score based on which the $\mathrm{k}^{\text {th }}$ category of assignment is to be assessed, then the expected score should not be less than the specified score. Therefore, by considering the above issues, the constraints on the targeted score may be $\mathrm{E}\left(\mathrm{T}_{\mathrm{k}^{*}} \mathrm{Y}_{\mathrm{k}}\right) \geq \mathrm{S}_{\mathrm{k}}$; which implies that $\sum_{i=1}^{m}\left(\sum_{j=1}^{n} j \cdot p_{i j}\right)\left(\sum_{j=1}^{n} q_{i j(2)}\right) * Y_{k} \geq S_{k} ;$ for $\mathrm{k}=1,2,3, \mathrm{r}$; 
Further, we consider a constraint that the overall decision score based on all ' $r$ ' categories. Let $\sum_{k=1}^{r} S_{k}=S$, then the constraint is $\sum_{k=1}^{r}\left(\sum_{i=1}^{m}\left(\sum_{j=1}^{n} j \cdot p_{i j}\right)\left(\sum_{j=1}^{n} q_{i j(2)}\right) * Y_{k}\right) \geq S$.

While achieving the objective, the decision maker has to observe the volatility score on decision making processes. Let $\mathrm{V}\left(\mathrm{T}_{\mathrm{k}}\right)$ be the variability of ' $\mathrm{k}$ 't ' type of one assignment. Then the variability for $\mathrm{Y}_{\mathrm{k}}$ number of assignments is $\mathrm{V}\left(\mathrm{T}_{\mathrm{k}^{*}} \mathrm{Y}_{\mathrm{k}}\right)$. Further, a healthy decision making procedure of $\mathrm{k}^{\text {th }}$ type requires the maximum restriction on the variability for $\mathrm{k}^{\text {th }}$ category of assignments. Let $\mathrm{V}_{\mathrm{k}}$ be the maximum desired variability for $\mathrm{Y}_{\mathrm{k}}$ number of assignments in the $\mathrm{k}^{\text {th }}$ category.

Therefore the overall variability for the $\mathrm{k}^{\text {th }}$ category should not exceed the specified level, i.e. $\mathrm{V}_{\mathrm{k}}$. Hence $\mathrm{V}\left(\mathrm{T}_{\mathrm{k}} * \mathrm{Y}_{\mathrm{k}}\right) \leq \mathrm{V}_{\mathrm{k}}$, which implies that

$$
\left(\sum_{i=1}^{m}\left(\sum_{j=1}^{n} j \cdot p_{i j}\right)^{2}\left(\sum_{j=1}^{n} q_{i j(2)}\right)-\sum_{i=1}^{m}\left(\sum_{j=1}^{n} j \cdot p_{i j}\right)\left(\sum_{j=1}^{n} q_{i j(2)}\right)^{2}\right) * Y_{k} \leq V_{k} ; \text { for } \mathrm{k}=1,2, \ldots . \text {.r. Further, we consider a }
$$

constraint that the overall variability on decision score based on all ' $r$ ' categories. Let $\sum_{k=1}^{r} V_{k}=V$, then the constraint is

$\sum_{k=1}^{r}\left(\sum_{i=1}^{m}\left(\sum_{j=1}^{n} j \cdot p_{i j}\right)^{2}\left(\sum_{j=1}^{n} q_{i j(2)}\right)-\sum_{i=1}^{m}\left(\sum_{j=1}^{n} j \cdot p_{i j}\right)\left(\sum_{j=1}^{n} q_{i j(2)}\right)^{2}\right) * Y_{k} \leq V$.

The decision variables under study are non negative. Hence $Y_{k} \geq 0$. Therefore the overall optimization programming problem is

Maximize $Z=\sum_{k=1}^{r}\left(\sum_{i=1}^{m}\left(\sum_{j=1}^{n} j \cdot p_{i j}\right)\left(\sum_{j=1}^{n} q_{i j(2)}\right)\right) * \mathrm{Y}_{k}$

subject to the constraints

$$
\begin{aligned}
& \sum_{i=1}^{m}\left(\sum_{j=1}^{n} j \cdot p_{i j}\right)\left(\sum_{j=1}^{n} q_{i j(2)}\right) * \mathrm{Y}_{k} \geq S_{k} ; \text { for } \mathrm{k}=1,2, \ldots \mathrm{r} ; \\
& \left(\sum_{i=1}^{m}\left(\sum_{j=1}^{n} j \cdot p_{i j}\right)^{2}\left(\sum_{j=1}^{n} q_{i j(2)}\right)-\sum_{i=1}^{m}\left(\sum_{j=1}^{n} j \cdot p_{i j}\right)\left(\sum_{j=1}^{n} q_{i j(2)}\right)^{2}\right) * \mathrm{Y}_{k} \leq V_{k} \quad \text { for } \mathrm{k}=1,2, \ldots . \mathrm{r} ; \\
& \sum_{k=1}^{r}\left(\sum_{i=1}^{m}\left(\sum_{j=1}^{n} j \cdot p_{i j}\right)\left(\sum_{j=1}^{n} q_{i j(2)}\right) * \mathrm{Y}_{k}\right) \geq S \\
& \sum_{k=1}^{r}\left(\sum_{i=1}^{m}\left(\sum_{j=1}^{n} j \cdot p_{i j}\right)^{2}\left(\sum_{j=1}^{n} q_{i j(2)}\right)-\sum_{i=1}^{m}\left(\sum_{j=1}^{n} j \cdot p_{i j}\right)\left(\sum_{j=1}^{n} q_{i j(2)}\right)^{2}\right) * \mathrm{Y}_{k} \leq V ; \\
& \text { and } \mathrm{Y}_{\mathrm{k}} \geq 0 ; \text { for } \mathrm{k}=1,2, \ldots \mathrm{r} ;
\end{aligned}
$$

\section{Numerical Illustration And Analysis}

The two models 2.1 and 2.2 are analyzed with a simulated numerical data sets. Extraction of decision variables, namely number of optimal decision assignments in each category can be obtained. All the above problems are comes under the category of Linear programming problems. Initial purpose of this chapter is to calculate the optimal function values and the values of decision variables. The next objective is to analyze the 
Managerial Decision scoring with Stochastic Programming on Selected Scaling Measures of Likert's

patterns of objective function values with its influencing factors such as $\mathrm{N}_{\mathrm{i}}, \mathrm{N}, \mathrm{P}_{\mathrm{ij}}, \mathrm{m}, \mathrm{n}, \mathrm{k}, \mathrm{r}, \mathrm{V}_{\mathrm{k}}, \mathrm{V}, \mathrm{S}_{\mathrm{k}}, \mathrm{S}, \mathrm{T}_{\mathrm{k}}, \mathrm{T}, \mathrm{W}_{\mathrm{i}}$, $\mathrm{C}_{\mathrm{k}}$. The study will provide detail understanding on the objective functions. The study also provides the guiding aspects on the constraints about their feasibilities and other related restrictions.

From Table-1 and Graph 1, it is observed that the objective value is an increasing function of $V_{1}, V_{2}, V_{3}$, $\mathrm{V}_{4}$, and $\mathrm{V}_{5}$ when other parameters are constant. Further the decision variable $\mathrm{X}_{1}, \mathrm{X}_{2}, \mathrm{X}_{3}, \mathrm{X}_{4}$, and $\mathrm{X}_{5}$ are increasing functions of $V_{1}, V_{2}, V_{3}, V_{4}$ and $V_{5}$ respectively and they are invariant with other complementary values of the respective Vi's for $i=1,2,3,4,5$.

From Table-2 and Graph-2, it is observed that the objective value is an increasing function of $V_{1}, V_{2}, V_{3}$, $\mathrm{V}_{4}$, and $\mathrm{V}_{5}$ when other parameters are constant. Further the decision variable $\mathrm{Y}_{1}, \mathrm{Y}_{2}, \mathrm{Y}_{3}, \mathrm{Y}_{4}$, and $\mathrm{Y}_{5}$ are increasing functions of $\mathrm{V}_{1}, \mathrm{~V}_{2}, \mathrm{~V}_{3}, \mathrm{~V}_{4}$ and $\mathrm{V}_{5}$ respectively and they are invariant with other complementary values of the respective Vi's for $\mathrm{i}=1,2,3,4,5$.

\section{References}

[1]. D Mather (1999), A framework for building spreadsheet based decision models; Journal of the Operational Research Society (1999) 50, 70-74.

[2]. Richard W.Kaz, Marc B. Carlange (1996), "Mixtures of stochastic processes: application to statistical downscaling"; Climate Research, Vol. 7: 185-193, 1996.

[3]. Thin-Yin Leong, Michelle L. F. Cheong (2008), "Teaching Business Modeling Using Spreadsheets" INFORMS Transactions on Education 9(1), pp. 20-34.

[4]. Tirupathi Rao Padi (2013), Stochastic Modeling on Likert's Scaling Measures; International Journal of Applied Mathematics \& Statistical Sciences; Vol. 2(5); 19-32

[5]. Tirupathi Rao Padi, Kiran Kumar Paidipati and C. Umasanker (2013), Stochastic Programming for Optimal Decision Making through Scaling Measures; International Journal of Management \& Information Technology; Vol. 6(1); 665-671.

[6]. Yuh-Yuan Guh, Rung-Wei Po, Kuo-Ren Lou (2009), "An Additive Scale Model for the Analytic Hierarchy Process"; International Journal of Information and Management Sciences; Vol. 20(2009), PP: 71-88

Table-1: Values of Decision variables and the optimal value of the objective function for varying values of other parameters(Model 2.1)

\begin{tabular}{|c|c|c|c|c|c|c|c|c|c|c|c|c|c|c|c|c|c|c|c|c|c|c|}
\hline \multicolumn{5}{|c|}{ Matrix } & $\mathrm{s}$ & $\mathbf{v}$ & $\mathrm{S}_{1}$ & $\mathrm{~S}_{2}$ & $\mathrm{~S}_{3}$ & $S_{4}$ & $S_{5}$ & $v_{1}$ & $\mathrm{~V}_{2}$ & $V_{3}$ & $\mathrm{~V}_{4}$ & $V_{5}$ & $\mathrm{X}_{1}$ & $\mathbf{X}_{2}$ & $\mathrm{X}_{3}$ & $\mathrm{X}_{4}$ & $X_{5}$ & $\mathrm{Z}$ \\
\hline 0 & 1 & 0 & 0 & 0 & 10 & 38 & 2 & 1 & 2 & 3 & 2 & 4 & 7 & 10 & 8 & 9 & 1.3265 & 1.6326 & 1.9387 & 1.7346 & 1.8367 & 25.408 \\
\hline 0 & 0 & 1 & 0 & 0 & & 45 & & & & & & 11 & & & & & 2.0408 & 1.6326 & 1.9387 & 1.7346 & 1.8367 & 27.551 \\
\hline 0 & 0 & 0 & 1 & 0 & & 49 & & & & & & 15 & & & & & 2.4489 & 1.6326 & 1.9387 & 1.7346 & 1.8367 & 28.775 \\
\hline 0 & 1 & 0 & 0 & 0 & & 54 & & & & & & 20 & & & & & 2.9591 & 1.6326 & 1.9387 & 1.7346 & 1.8367 & 30.306 \\
\hline 0 & 0 & 0 & 1 & 0 & & 57 & & & & & & 23 & & & & & 3.2653 & 1.6326 & 1.9387 & 1.7346 & 1.8367 & 31.224 \\
\hline 0 & 1 & 0 & 0 & 0 & 10 & 43 & 2 & 1 & 2 & 3 & 2 & 4 & 12 & 10 & 8 & 9 & 1.3265 & 2.1428 & 1.9387 & 1.7346 & 1.8367 & 26.938 \\
\hline 0 & 0 & 1 & 0 & 0 & & 48 & & & & & & & 17 & & & & 1.3265 & 2.6530 & 1.9387 & 1.7346 & 1.8367 & 28.469 \\
\hline 0 & 0 & 0 & 1 & 0 & & 53 & & & & & & & 22 & & & & 1.3265 & 3.1632 & 1.9387 & 1.7346 & 1.8367 & 30.000 \\
\hline 0 & 1 & 0 & 0 & 0 & & 58 & & & & & & & 27 & & & & 1.3265 & 3.6734 & 1.9387 & 1.7346 & 1.8367 & 31.531 \\
\hline 0 & 0 & 0 & 1 & 0 & & 63 & & & & & & & 32 & & & & 1.3265 & 4.1836 & 1.9387 & 1.7346 & 1.8367 & 33.061 \\
\hline 0 & 1 & 0 & 0 & 0 & 10 & 42 & 2 & 1 & 2 & 3 & 2 & 4 & 7 & 14 & 8 & 9 & 1.3265 & 1.6326 & 2.3469 & 1.7346 & 1.8367 & 26.633 \\
\hline 0 & 0 & 1 & 0 & 0 & & 46 & & & & & & & & 18 & & & 1.3265 & 1.6326 & 2.7551 & 1.7346 & 1.8367 & 27.857 \\
\hline 0 & 0 & 0 & 1 & 0 & & 50 & & & & & & & & 22 & & & 1.3265 & 1.6326 & 3.1632 & 1.7346 & 1.8367 & 29.082 \\
\hline 0 & 1 & 0 & 0 & 0 & & 54 & & & & & & & & 26 & & & 1.3265 & 1.6326 & 3.5714 & 1.7346 & 1.8367 & 30.306 \\
\hline 0 & 0 & 0 & 1 & 0 & & 58 & & & & & & & & 30 & & & 1.3265 & 1.6326 & 3.9795 & 1.7346 & 1.8367 & 31.531 \\
\hline 0 & 1 & 0 & 0 & 0 & 10 & 41 & 2 & 1 & 2 & 3 & 2 & 4 & 7 & 10 & 11 & 9 & 1.3265 & 1.6326 & 1.9387 & 2.0408 & 1.8367 & 26.326 \\
\hline 0 & 0 & 1 & 0 & 0 & & 44 & & & & & & & & & 14 & & 1.3265 & 1.6326 & 1.9387 & 2.3469 & 1.8367 & 27.245 \\
\hline 0 & 0 & 0 & 1 & 0 & & 47 & & & & & & & & & 17 & & 1.3265 & 1.6326 & 1.9387 & 2.6530 & 1.8367 & 28.163 \\
\hline 0 & 1 & 0 & 0 & 0 & & 50 & & & & & & & & & 20 & & 1.3265 & 1.6326 & 1.9387 & 2.9591 & 1.8367 & 29.082 \\
\hline 0 & 0 & 0 & 1 & 0 & & 53 & & & & & & & & & 23 & & 1.3265 & 1.6326 & 1.9387 & 3.2653 & 1.8367 & $\mathbf{3 0 . 0 0 0}$ \\
\hline 0 & 1 & 0 & 0 & 0 & 10 & 40 & 2 & 1 & 2 & 3 & 2 & 4 & 7 & 10 & 8 & 11 & 1.3265 & 1.6326 & 1.9387 & 1.7346 & 2.0408 & 26.020 \\
\hline 0 & 0 & 1 & 0 & 0 & & 42 & & & & & & & & & & 13 & 1.3265 & 1.6326 & 1.9387 & 1.7346 & 2.2448 & 26.633 \\
\hline 0 & 0 & 0 & 1 & 0 & & 44 & & & & & & & & & & 15 & 1.3265 & 1.6326 & 1.9387 & 1.7346 & 2.4489 & 27.245 \\
\hline 0 & 1 & 0 & 0 & 0 & & 46 & & & & & & & & & & 17 & 1.3265 & 1.6326 & 1.9387 & 1.7346 & 2.6530 & 27.857 \\
\hline 0 & 0 & 0 & 1 & 0 & & 48 & & & & & & & & & & 19 & 1.3265 & 1.6326 & 1.9387 & 1.7346 & 2.8571 & 28.469 \\
\hline
\end{tabular}


Managerial Decision scoring with Stochastic Programming on Selected Scaling Measures of Likert's

Table-2: Values of Decision variables and the optimal value of the objective function for varying values of other parameters (Model 2.2.)

\begin{tabular}{|c|c|c|c|c|c|c|c|c|c|c|c|c|c|c|c|c|c|c|c|c|c|c|}
\hline \multicolumn{5}{|c|}{ Matrix } & $\mathrm{S}$ & $\mathrm{V}$ & $\mathrm{S}_{1}$ & $S_{2}$ & $\mathrm{~S}_{3}$ & $S_{4}$ & $\mathrm{~S}_{5}$ & $\mathrm{~V}_{1}$ & $\mathbf{V}_{2}$ & $V_{3}$ & $V_{4}$ & $V_{5}$ & $\mathbf{Y}_{1}$ & $\mathbf{Y}_{2}$ & $\mathbf{Y}_{3}$ & $\mathbf{Y}_{4}$ & $Y_{5}$ & $\mathrm{Z}$ \\
\hline 0 & 1 & 0 & 0 & 0 & 10 & 38 & 2 & $I$ & 2 & 3 & 2 & 4 & 7 & 10 & 8 & 9 & 5.00 & 8.75 & 12.50 & 10.00 & 11.25 & 142.50 \\
\hline 0 & 0 & 1 & 0 & 0 & & 45 & & & & & & 11 & & & & & 13.75 & 8.75 & 12.50 & 10.00 & 11.25 & 168.25 \\
\hline 0 & 0 & 0 & 1 & 0 & & 49 & & & & & & 15 & & & & & 18.75 & 8.75 & 12.50 & 10.00 & 11.25 & 183.75 \\
\hline 0 & 1 & 0 & 0 & 0 & & 54 & & & & & & 20 & & & & & 25.00 & 8.75 & 12.50 & 10.00 & 11.25 & 202.50 \\
\hline 0 & 0 & 0 & 1 & 0 & & 57 & & & & & & 23 & & & & & 28.75 & 8.75 & 12.50 & 10.00 & 11.25 & 213.75 \\
\hline 0 & 1 & 0 & 0 & 0 & 10 & 43 & 2 & 1 & 2 & 3 & 2 & 4 & 12 & 10 & 8 & 9 & 5.00 & 15.00 & 12.50 & 10.00 & 11.25 & 161.25 \\
\hline 0 & 0 & 1 & 0 & 0 & & 48 & & & & & & & 17 & & & & 5.00 & 21.25 & 12.50 & 10.00 & 11.25 & 180.00 \\
\hline 0 & 0 & 0 & 1 & 0 & & 53 & & & & & & & 22 & & & & 5.00 & 27.50 & 12.50 & 10.00 & 11.25 & 198.75 \\
\hline 0 & 1 & 0 & 0 & 0 & & 58 & & & & & & & 27 & & & & 5.00 & 33.75 & 12.50 & 10.00 & 11.25 & 217.50 \\
\hline 0 & 0 & 0 & 1 & 0 & & 63 & & & & & & & 32 & & & & 5.00 & 40.00 & 12.50 & 10.00 & 11.25 & 236.25 \\
\hline 0 & 1 & 0 & 0 & 0 & 10 & 42 & 2 & 1 & 2 & 3 & 2 & 4 & 7 & 14 & 8 & 9 & 5.00 & 8.75 & 17.50 & 10.00 & 11.25 & 157.50 \\
\hline 0 & 0 & 1 & 0 & 0 & & 46 & & & & & & & & 18 & & & 5.00 & 8.75 & 22.50 & 10.00 & 11.25 & 172.50 \\
\hline 0 & 0 & 0 & 1 & 0 & & 50 & & & & & & & & 22 & & & 5.00 & 8.75 & 27.50 & 10.00 & 11.25 & 187.50 \\
\hline 0 & 1 & 0 & 0 & 0 & & 54 & & & & & & & & 26 & & & 5.00 & 8.75 & 32.50 & 10.00 & 11.25 & 202.50 \\
\hline 0 & 0 & 0 & 1 & 0 & & 58 & & & & & & & & 30 & & & 5.00 & 8.75 & 37.50 & 10.00 & 11.25 & 217.50 \\
\hline 0 & 1 & 0 & 0 & 0 & 10 & 41 & 2 & 1 & 2 & 3 & 2 & 4 & 7 & 10 & 11 & 9 & 5.00 & 8.75 & 12.50 & 13.75 & 11.25 & 153.75 \\
\hline 0 & 0 & 1 & 0 & 0 & & 44 & & & & & & & & & 14 & & 5.00 & 8.75 & 12.50 & 17.50 & 11.25 & 165.00 \\
\hline 0 & 0 & 0 & 1 & 0 & & 47 & & & & & & & & & 17 & & 5.00 & 8.75 & 12.50 & 21.25 & 11.25 & 176.25 \\
\hline 0 & 1 & 0 & 0 & 0 & & 50 & & & & & & & & & 20 & & 5.00 & 8.75 & 12.50 & 25.00 & 11.25 & 187.50 \\
\hline 0 & 0 & 0 & 1 & 0 & & 53 & & & & & & & & & 23 & & 5.00 & 8.75 & 12.50 & 28.75 & 11.25 & 198.75 \\
\hline 0 & 1 & 0 & 0 & 0 & 10 & 40 & 2 & 1 & 2 & 3 & 2 & 4 & 7 & 10 & 8 & 11 & 5.00 & 8.75 & 12.50 & 10.00 & 13.75 & 150.00 \\
\hline 0 & 0 & 1 & 0 & 0 & & 42 & & & & & & & & & & 13 & 5.00 & 8.75 & 12.50 & 10.00 & 16.25 & 157.50 \\
\hline 0 & 0 & 0 & 1 & 0 & & 44 & & & & & & & & & & 15 & 5.00 & 8.75 & 12.50 & 10.00 & 18.75 & 165.00 \\
\hline 0 & 1 & 0 & 0 & 0 & & 46 & & & & & & & & & & 17 & 5.00 & 8.75 & 12.50 & 10.00 & 21.25 & 172.50 \\
\hline 0 & 0 & 0 & 1 & 0 & & 48 & & & & & & & & & & 19 & 5.00 & 8.75 & 12.50 & 10.00 & 23.75 & 180.00 \\
\hline
\end{tabular}

MODEL : 2.1

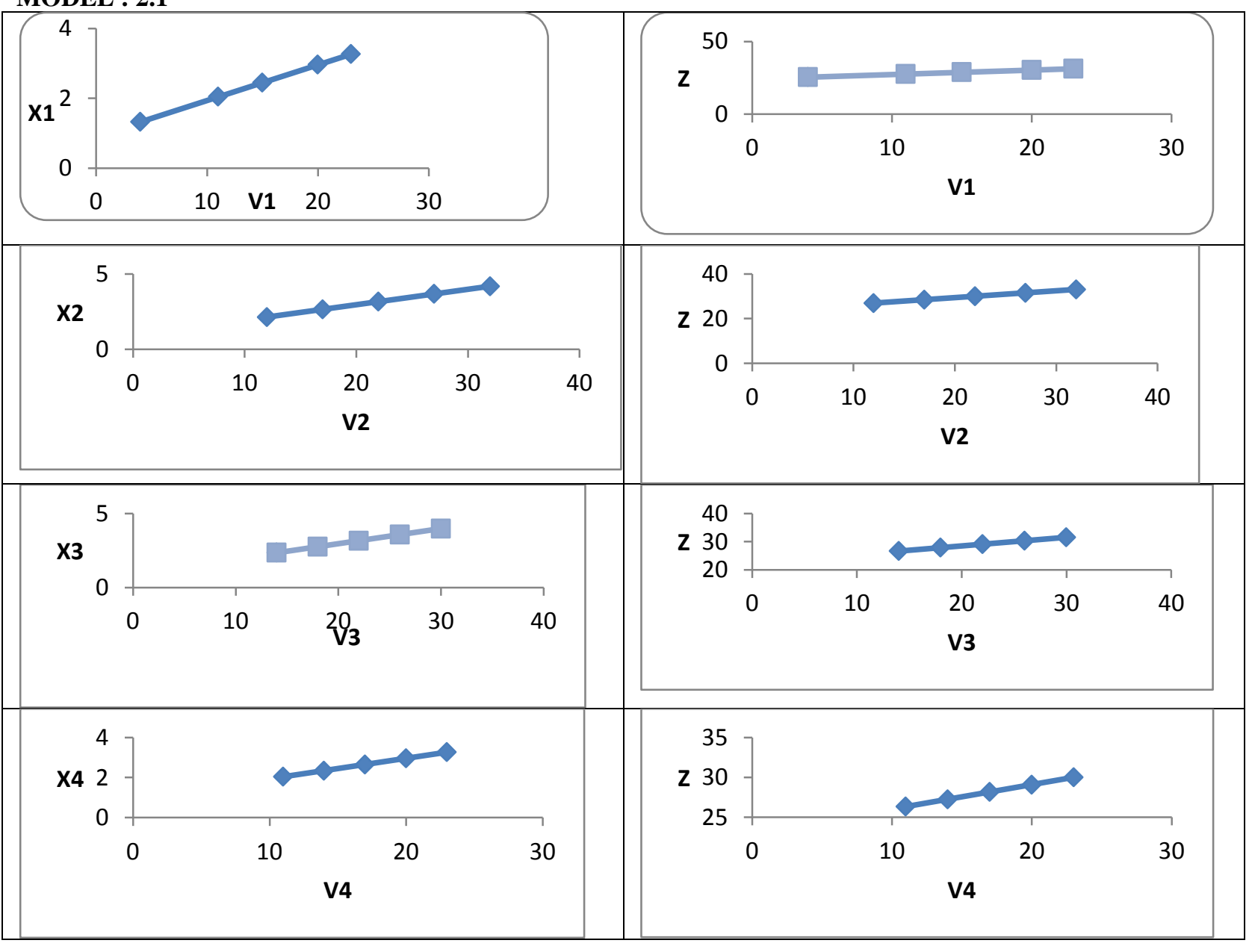


Managerial Decision scoring with Stochastic Programming on Selected Scaling Measures of Likert's

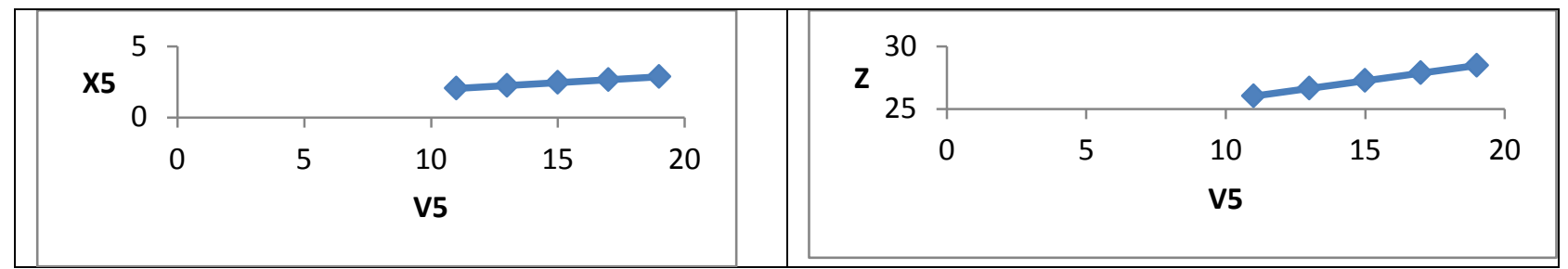

MODEL : 2.2

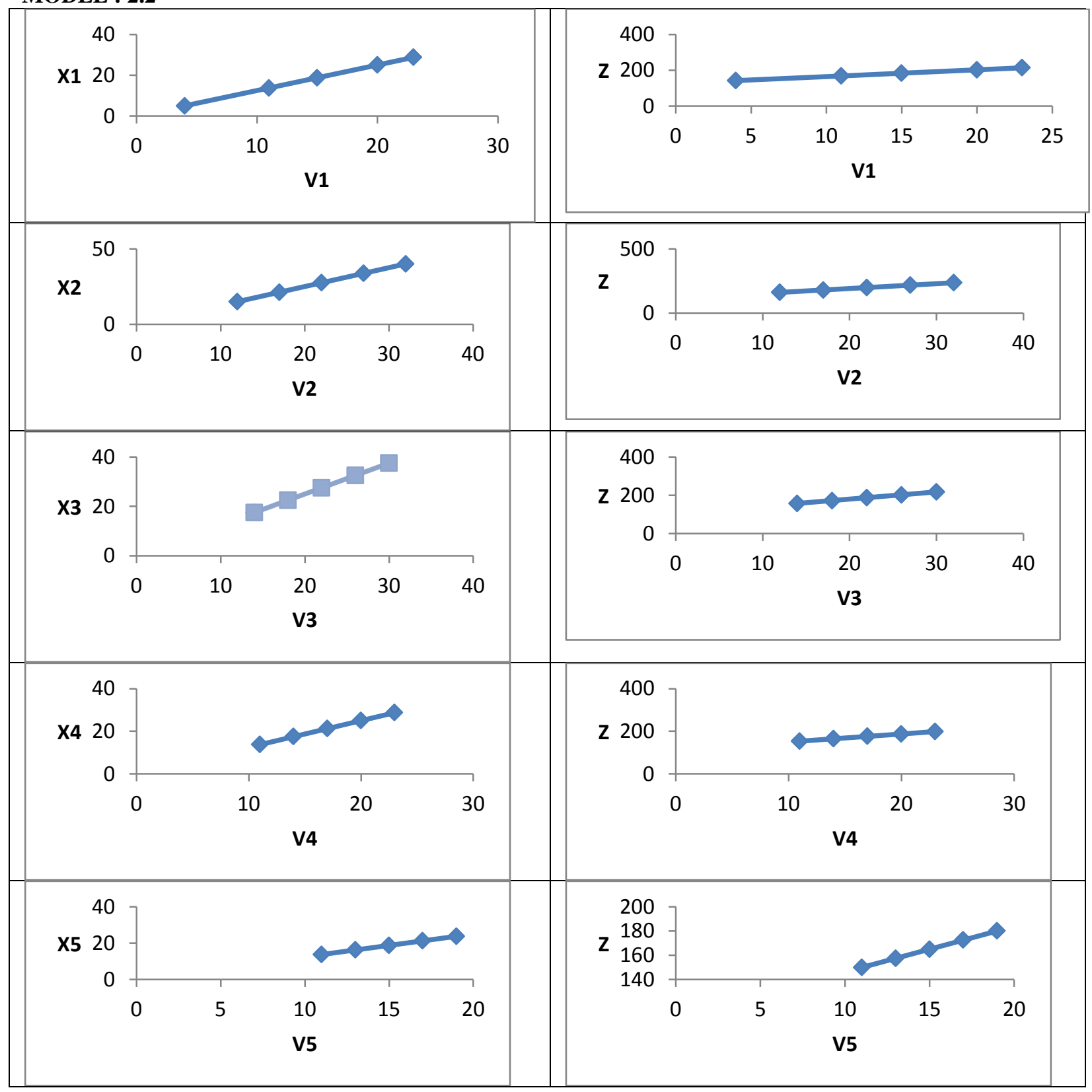

